\title{
Clopidogrel Resistance in Neurovascular Stenting: Correlations between Light Transmission Aggregometry, VerifyNow, and the Multiplate
}

\author{
N. Flechtenmacher, F. Kämmerer, R. Dittmer, U. Budde, P. Michels, J. Röther, and B. Eckert
}

\begin{abstract}
BACKGROUND AND PURPOSE: Clopidogrel resistance is blamed for thromboembolic complications in neurovascular stent placement. Platelet-function assays are weakly standardized. The aim of this study was to correlate the results of 3 different platelet-inhibition measurements (from light transmission aggregometry, the VerifyNow P2Y12 test, and the Multiplate analyzer) and their relation to periprocedural thromboembolic complications in elective neurovascular stent placement.
\end{abstract}

MATERIALS AND METHODS: Clopidogrel resistance was determined on the day of the intervention according to predefined platelet reactivity cutoff values. All 3 tests were performed in 103 consecutive neurovascular stent-placement procedures in 97 patients (extracranial, $n=77$; intracranial, $n=26)$.

RESULTS: The clopidogrel resistance rates were 47.6\% (light transmission aggregometry), 50.5\% (VerifyNow), and 35.9\% (Multiplate). In 67\% of the patients, clopidogrel resistance was present according to at least one method. The correlations of qualitative results that classified a patient as responsive or resistant to clopidogrel were $67.9 \%$ for light transmission aggregometry with VerifyNow, $77.7 \%$ for light transmission aggregometry with the Multiplate, and $66 \%$ for VerifyNow with the Multiplate. Periprocedural thromboembolic complications ( $n=9$ ) occurred more frequently in patients who were determined by all 3 methods to be clopidogrel resistant. The difference was most pronounced with light transmission aggregometry (complication rates, 14.4\% [clopidogrel-resistant patients] vs 3.7\% [clopidogrelresponsive patients]). Sensitivity and specificity rates of clopidogrel resistance in relation to embolic complications were, respectively, $78 \%$ and 55\% for light transmission aggregometry, $67 \%$ and $51 \%$ for VerifyNow, and $44 \%$ and $67 \%$ for the Multiplate.

CONCLUSIONS: Clopidogrel resistance is a frequent finding in patients who undergo neurovascular stent placement. The correlations among the different testing methods are only modest and differ considerably. Light transmission aggregometry results seem to correlate with thromboembolic complications more accurately than with VerifyNow and Multiplate point-of-care methods.

ABBREVIATION: LTA = light transmission aggregometry

$\mathrm{n}$ neurovascular procedures, especially the placement of a stent into an extracranial or intracranial artery, clopidogrel resistance is associated with an increased risk of thromboembolic complications. ${ }^{1-5}$ In elective neurovascular stent placement, preprocedural testing of platelet inhibition is increasingly performed to identify patients with clopidogrel resistance. However, test result standards for clopidogrel resistance have not been established, and strong clinical evidence that supports the idea that the results of such tests definitively alter clinical outcomes is still

Received December 22, 2014; accepted after revision March 3, 2015.

From the Departments of Neuroradiology (N.F., F.K., B.E.) and Neurology (P.M., J.R.) Asklepios Klinik Altona, Hamburg, Germany; and Department of Laboratory Medicine (R.D., U.B.), Medilys Laboratory Company, Hamburg, Germany.

Please address correspondence to B. Eckert, Prof Dr Med, Department of Neuroradiology Asklepios Klinik Altona, Abteilung für Neuroradiologie, Paul-Ehrlich-Str 1, 22763 Hamburg, Germany; e-mail: b.eckert@asklpios.com.

http://dx.doi.org/10.3174/ajnr.A4388 lacking. Light transmission aggregometry (LTA) is considered the criterion-standard test method, but standard values have not yet been established. LTA requires a preparation time of 2 hours, whereas point-of-care methods such as the VerifyNow P2Y12 assay (Accumetrics, San Diego, California) and impedance aggregometry using a Multiplate analyzer (Dynabyte, Munich, Germany) offer periprocedural test results within minutes in the operating suite. ${ }^{6}$

A study comparing LTA with point-of-care measurements in percutaneous coronary intervention has shown only modest agreement among the different tests. ${ }^{7}$ In neurovascular procedures, clopidogrel resistance has been investigated with various methods. However, a comparison of different test methods to determine the correlation of their results with the occurrence of embolic complications has not yet been performed in the clinical context of neurovascular stent placement.

AJNR Am J Neuroradiol 36:1953-58 Oct 2015 www.ajnr.org 
The purpose of this study was to determine the correlation of platelet inhibition with clopidogrel according to 3 different testing methods and the association of clopidogrel resistance with thromboembolic complications in elective neurovascular stent placement.

\section{MATERIALS AND METHODS}

Between September 2011 and August 2012, all consecutive patients who were undergoing elective intracranial or extracranial stent placement for atherosclerotic stenosis or stent-assisted coiling of intracranial aneurysms were included in this prospective study. Patients who underwent extracranial stent placement received local anesthesia, and those who underwent intracranial stent placement were intubated and received general anesthesia. Each patient received $100 \mathrm{mg}$ of aspirin and $75 \mathrm{mg}$ of clopidogrel daily for 5 days before the procedure $(n=80)$ or a loading dose of $600 \mathrm{mg}$ of clopidogrel the day before the procedure $(n=23)$. Medication lists of the patients were reviewed before initiating clopidogrel to ensure that they were not taking any drugs that would potentially interact (eg, cytochrome P450 2C19 inhibitors, including proton-pump inhibitors), because that would have affected the results. The study was approved by the Ärztekammer Hamburg ethical review board, and written informed consent was obtained from each patient. Clopidogrel resistance was analyzed in each patient on the day of the procedure by using LTA (induced by $2 \mu \mathrm{mol} / \mathrm{L}$ adenosine diphosphate), the VerifyNow P2Y12 assay, and impedance aggregometry using a Multiplate analyzer. LTA is a method that measures adenosine diphosphate-induced platelet aggregation in platelet-rich plasma by changes in light transmittance. Platelet-rich plasma is acquired by centrifuging citrate-anticoagulated whole blood, which requires a preparation time of 2 hours. The interpretation of the test results requires standardization and a trained laboratory staff. The VerifyNow system is a turbidimetric-based optical detection system that measures platelet-induced aggregation as an increase in light transmittance with citrate-anticoagulated whole blood. The VerifyNow P2Y12 assay is a fast, standardized, point-of-care test that does not require any special training for its performance. The Multiplate analyzer detects platelet aggregation by measuring impedance changes. Hirudin-anticoagulated whole blood is pipetted into a test cell. Aggregation starts by adding the agonist adenosine diphosphate. Pipetting is performed by an attached electronic pipette. No trained staff is needed to perform the test. Clopidogrel resistance was defined as follows: for the VerifyNow P2Y12 assay, $>236$ P2Y12 reaction units; for LTA, $>40 \%$ of maximal aggregation and disaggregation curve; and for the Multiplate, $>40-\mathrm{U}$ area under the curve value. If 2 of the 3 methods indicated resistance, the clopidogrel dose was increased to $150 \mathrm{mg}$ daily after the procedure. Follow-up platelet-activity testing was not performed. Clopidogrel was maintained for 2 months after the extracranial procedures and 3 months after the intracranial stent-placement procedures; aspirin was continued long term. The stent-placement procedures were performed by 1 of 2 neurointerventionists (A.L. or B.E.) with $>15$ years' experience in neurointerventional procedures, both of whom were certified in the stent arm of the German Stent-Protected Angioplasty versus Carotid Endarterectomy (SPACE) study. Neurologic examination was performed on
Table 1: Incidence of clopidogrel resistance

\begin{tabular}{lll}
\hline \multicolumn{1}{c}{ Clopidogrel Status } & $\boldsymbol{n}$ & $\%$ \\
\hline Resistant according to: & & \\
$\geq 1$ method & 69 & 67 \\
$\geq 2$ methods & 43 & 41.9 \\
All 3 methods & 24 & 23.3 \\
Clopidogrel responsive according to all 3 methods & 34 & 33 \\
\hline
\end{tabular}

each patient promptly after the intervention and before discharge by an independent neurologist. Each patient was monitored on a stroke unit certified by the German Stroke Society for at least 24 hours. In case of a new neurologic deficit, MR imaging was performed. A thromboembolic complication was defined as transient or persistent neurologic deficits associated with new DWI lesions found on MR imaging. NIHSS and the mRS were used to grade and follow up on neurologic deficits. After 3 months, clinical follow-up was accomplished by a structured telephone interview.

All statistical tests were performed with the use of SAS software version 9.3 (SAS Institute, Cary, North Carolina). For correlations between the continuous values of the 3 test methods, Spearman rank-order correlation $(\rho)$ was used. A perfect monotonic relationship is represented by a $\rho$ value of 1 or -1 . The clopidogrel resistance status in each method was entered in a multiple logistic regression model.

The sensitivity, specificity, positive predictive value, and negative predictive value of the 3 methods relating to the occurrence of thromboembolic events were calculated by building contingency tables.

Differences between categoric variables were evaluated with the $\chi^{2}$ test or with the Fisher exact test in case of small expected cell frequencies. All $P$ values were 2 -sided. For all the statistical tests, a $P$ value of $<.05$ was considered significant.

\section{RESULTS}

During the study period, 107 patients underwent neurovascular stent placement. Ten patients were excluded because of insufficient laboratory data or different antiplatelet therapy. The data analysis included 103 procedures in 97 patients. The procedures were stent placements for extracranial stenosis $(n=77)$ or intracranial stenosis $(n=16)$ and stent-assisted intracranial aneurysm coilings $(n=10)$. The mean patient age was 67.2 years; there were 64 male and 33 female patients.

Clopidogrel resistance was detected with LTA in 49 (47.6\%) measurements, with VerifyNow in $52(50.5 \%)$ measurements, and with the Multiplate in 35 (34\%) measurements. The incidences of clopidogrel resistance in the 3 tests are listed in Table 1. In $33 \%$ of the measurements, all 3 tests indicated sufficient platelet inhibition; in the remaining two-thirds of the cohort, clopidogrel resistance was found in at least 1 of the 3 methods, and in $42 \%$, clopidogrel resistance was found in 2 of 3 assays. Concordant clopidogrel resistance in all 3 methods was seen in $23 \%$ of the measurements. According to the study protocol, 39 patients (43 procedures) received $150 \mathrm{mg}$ of clopidogrel after the procedure.

The individual test results for each method are shown in Fig 1.

Correlation scatter plots of the test results are shown in Fig 2. The statistical analysis revealed significant correlations among the quantitative values for LTA with the Multiplate $(\rho=0.52 ; P=$ 

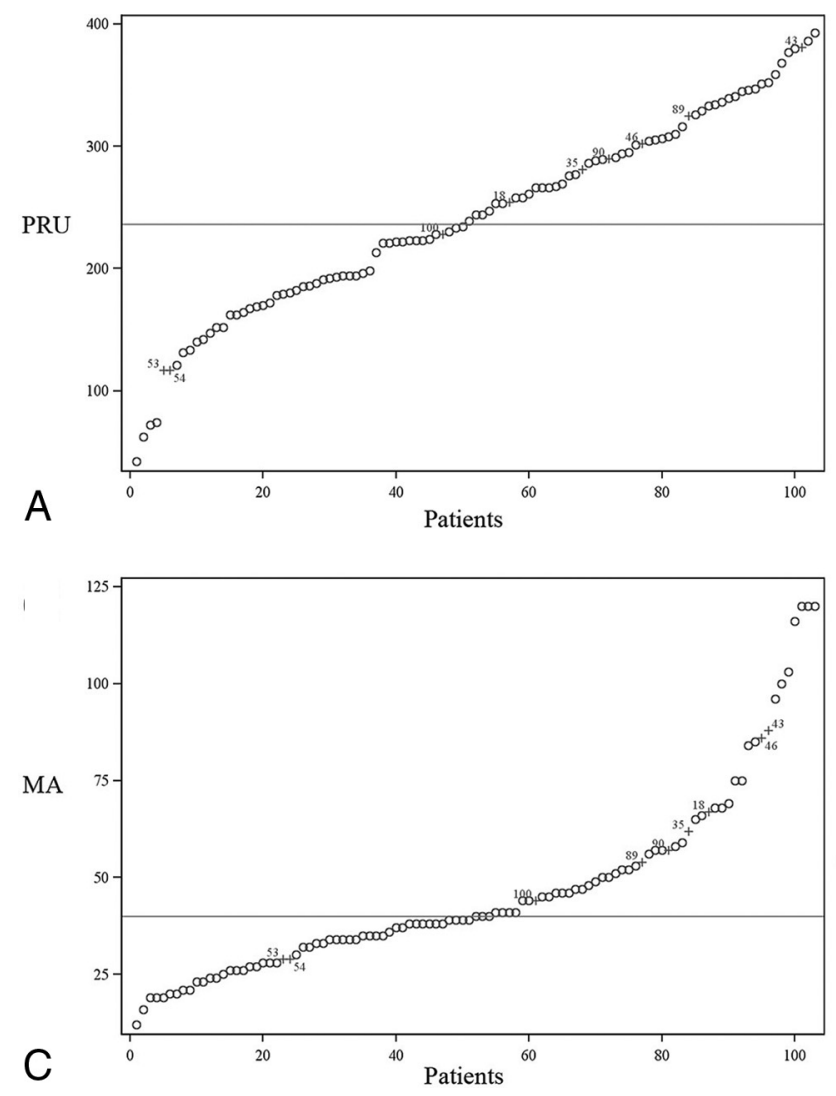

$.0001)$, LTA with VerifyNow $(\rho=0.33 ; P=.0008)$, and VerifyNow with the Multiplate $(\rho=0.28 ; P=.0041)$. The correlations of the qualitative classification of a patient as clopidogrel responsive or resistant were $67.9 \%$ for LTA with VerifyNow, $77.7 \%$ for LTA with the Multiplate, and $66 \%$ for VerifyNow with the Multiplate.

Nine thromboembolic events that led to major stroke (1 patient), minor stroke (4 patients), or TIA (4 cases) occurred during the interventions. Thromboembolic complications occurred in 2 cases of stent-assisted aneurysm coiling, in 2 cases of extracranial vertebral stent placement, and in 5 cases of extracranial carotid stent placement. During the stent placements for intracranial stenosis, no thromboembolic complications occurred. In all 4 patients with transient symptoms, postprocedural MR imaging detected new DWI lesions. Additional TIAs without DWI lesions did not occur. No delayed thromboembolic complications (before discharge) occurred in any patient.

One patient who experienced thromboembolic procedural TIAs in 2 separate treatments of a symptomatic extracranial ICA stenosis and a vertebral artery-origin stenosis was classified as clopidogrel responsive by all 3 test methods in both stent-placement procedures (Nr.53 and Nr.54 in Figures). In this patient, no hematologic or anatomic abnormalities were found. In the remaining 7 complications, clopidogrel resistance was identified in all cases by LTA, in 6 cases by VerifyNow, and in 4 cases by the Multiplate. The correlations of the qualitative test results of each method with the occurrence of thromboembolic complications are listed in Table 2. Results of the statistical analysis of the qualitative test results in relation to thromboembolic complications (yes/no) are shown in Table 2.

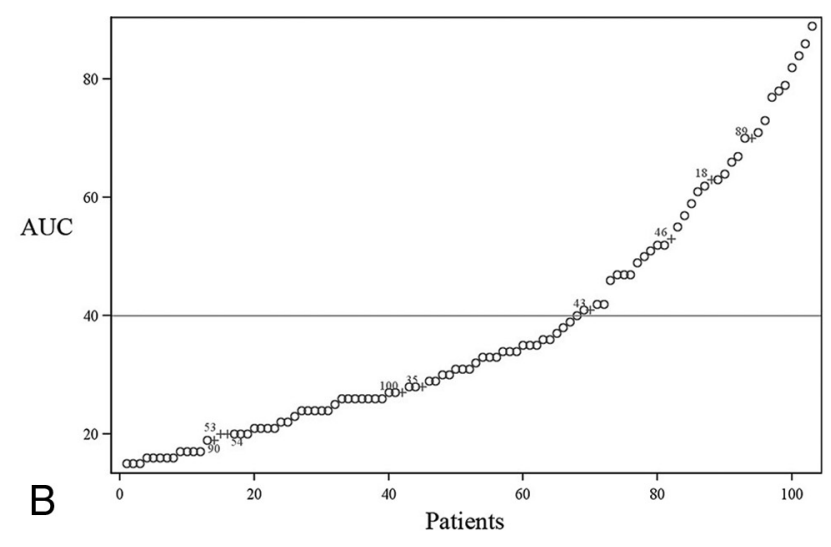

FIG 1. Individual test results of VerifyNow $(A)$, the Multiplate $(B)$, and LTA (C). The line parallel to the $x$-axis marks the particular cutoff value that has been used to discriminate between clopidogrel resistance and clopidogrel response. Procedures in which an embolic complication occurred are indicated by plus signs. Embolic complications Nr.53 and Nr.54 occurred in a single patient with 2 subsequent treatments of a symptomatic ICA and vertebral artery stenosis, respectively. PRU indicates P2Y12 reaction units; MA, maximal aggregation; AUC, area under the curve.

Follow-up data were available after 3 months for 75 patients (81 stent-placement procedures), including all 39 patients whose dosage of clopidogrel was doubled. Follow-up telephone interviews did not reveal any medication-noncompliance issues. During the follow-up period, 1 ipsilateral stroke and 3 ipsilateral TIAs were detected. One postdischarge stroke occurred after ICA stent placement in a patient (Nr.100) who was identified as clopidogrel resistant by LTA alone and treated with $75 \mathrm{mg}$ of clopidogrel after the procedure. Three postdischarge TIAs occurred after extracranial ICA stent placement. All 3 patients were identified as clopidogrel resistant according to all 3 tests and were treated with 150 $\mathrm{mg}$ of clopidogrel after the procedure. A symptomatic bleeding complication occurred in 1 patient suffering epistaxis and required embolization treatment; the patient recovered completely. Neither stent thrombosis nor intracerebral bleeding was detected in any patient.

\section{DISCUSSION}

To our knowledge, this is the first study to correlate 3 different clopidogrel-reactivity assays in the clinical context of elective neurovascular stent placement. In $67 \%$ of the patients, at least one test method revealed clopidogrel resistance. The clopidogrel-responsive status differed among the different methods for a considerable number of patients. Embolic complications occurred more frequently in patients with clopidogrel resistance determined by all 3 types of tests. The LTA results revealed a more accurate correlation of clopidogrel resistance and thromboembolic complications than the VerifyNow and Multiplate point-of-care methods.

The rates of clopidogrel resistance differed among the Multiplate (36\%), LTA (48\%), and VerifyNow (50\%) methods. These 


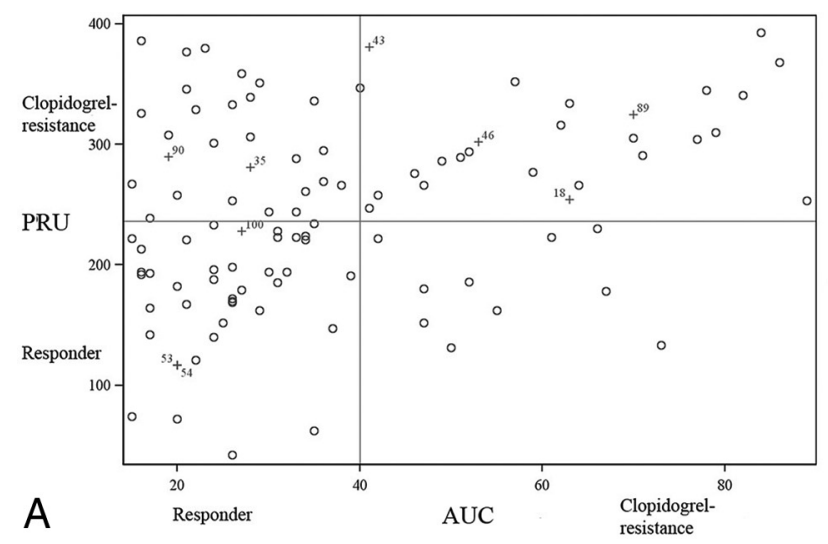

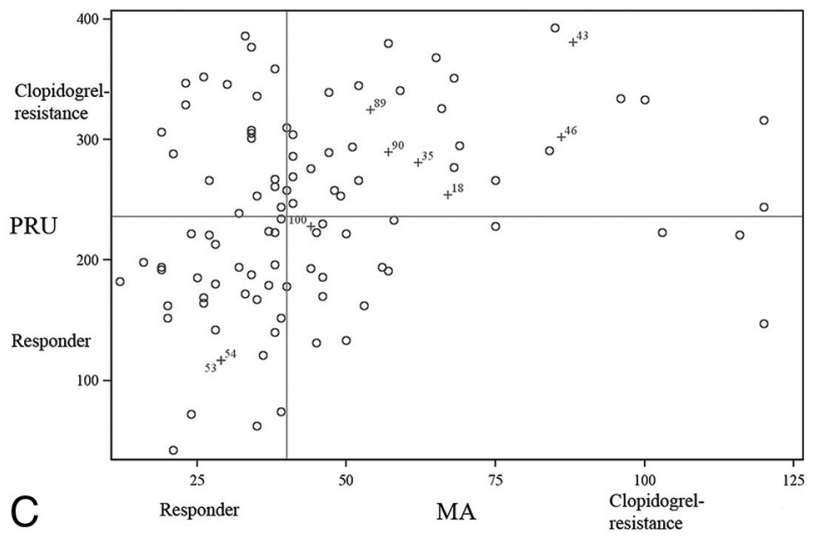

data are in accordance with those of previous studies, which have indicated clopidogrel-resistance rates from $37 \%$ up to $52 \%$ in patients who were undergoing cerebrovascular stent placement. ${ }^{2,5}$ The clopidogrel dosing schedule in our study was $75 \mathrm{mg}$ daily for 5-7 days before the intervention or a 600-mg loading dose on the day before the intervention. A loading dose of clopidogrel is usually given just 1 day before the procedure. Some centers, especially cardiology, recommend a loading dose of 300-600 mg of clopidogrel even 5 days before intervention. The relatively low doses may explain the high percentage of clopidogrel-resistant patients in comparison with that found in cardiology studies. Analysis of the correlation of different test methods was performed in one percutaneous coronary intervention study that compared LTA, VerifyNow, and a vasodilator-stimulated phosphoprotein phosphorylation assay. The incidence of clopidogrel resistance varied from $16 \%$ up to $39 \%$ in the patients. The level of agreement between the assays was in the moderate-to-poor range, with Spearman correlation coefficients between 0.60 and $0.86 .^{7}$ Statistical analysis of the quantitative results in our study revealed significant but poor correlations between the different types of tests, with Spearman correlation coefficients between 0.28 and 0.52 . In addition, the correlation of the qualitative classifications of a patient as clopidogrel responsive or resistant was poor in our study and ranged between $66 \%$ and $78 \%$ with the different measurements. In accordance with the percutaneous coronary intervention study by Gaglia et $\mathrm{al}^{7}$ the results of our study underline the fact that results of the different tests do not agree in a notable number of patients.

Several studies have found a strong correlation of insufficient clopidogrel-related platelet inhibition and an increased risk of

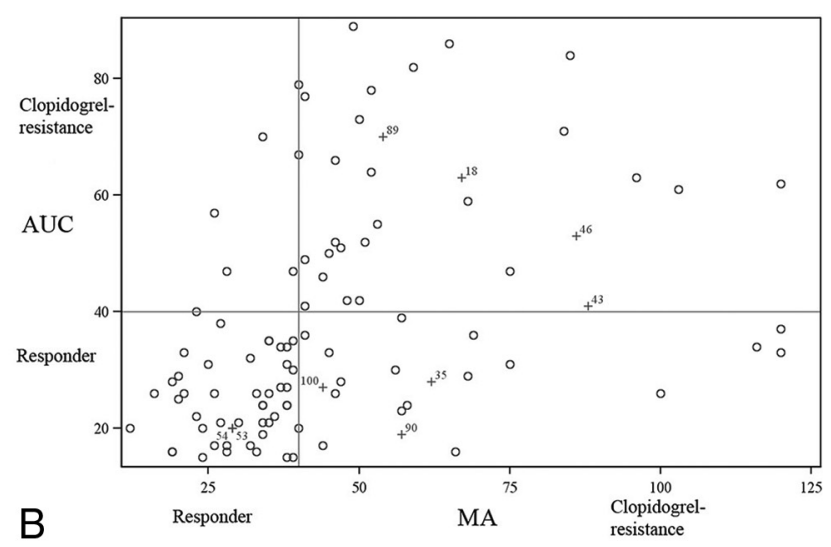

FIG 2. Correlation scatterplots of individual results obtained with VerifyNow versus the Multiplate (A), the Multiplate versus LTA (B), and VerifyNow versus LTA $(C)$. Lines parallel to the axes mark the particular cutoff values. The upper-right and lower-left quadrants contain values with agreement in regard to the definition of clopidogrel resistance. Procedures in which an embolic complication occurred are indicated by plus signs.

thromboembolic events in supra-aortic stent placement and in cerebral aneurysm coiling. ${ }^{1,3-5,8}$ In line with the results of previous studies, we found increased complication rates in patients with clopidogrel resistance as determined by all 3 methods, but because of the small number of patients, the difference was not statistically significant. In patients who were deemed clopidogrel resistant by LTA, the complication rate was $14.3 \%$ (vs $3.7 \%$ of clopidogrel-responsive patients). The discrepancies in complication rates between clopidogrel-resistant and -responsive patients were less convincing with the VerifyNow ( $11.5 \%$ vs $5.9 \%$, respectively) and Multiplate (11.4\% vs $7.5 \%$, respectively) methods. Hence, the statistical correlation of clopidogrel reactivity and the occurrence of embolic complications indicates a higher sensitivity for LTA (78\%) than for VerifyNow (67\%) and Multiplate (44\%) testing.

In the present study, 1 patient who suffered a procedural thromboembolic TIA in 2 separate treatments of a symptomatic extracranial ICA stenosis and a vertebral artery-origin stenosis was classified as clopidogrel responsive by all 3 test methods in both stent-placement procedures, indicating that clopidogrel resistance is a major, but not the exclusive, factor of thromboembolic complications. In the remaining 7 thromboembolic complications, LTA identified clopidogrel resistance in all cases, VerifyNow in 6 cases, and the Multiplate in 4 cases.

The pharmacologic response of the P2Y12 receptor antagonists such as clopidogrel strongly depends on cytochrome P450 genetic polymorphism. Carriers of reduced-function CYP2C19 alleles have significantly lower levels of active metabolite, resulting in diminished platelet inhibition. ${ }^{9,10}$ The pharmacodynamic process of converting the predrug into the active metabolite dif- 
Table 2: Correlation of clopidogrel test results and thromboembolic events

\begin{tabular}{lcccccc}
\hline Method & $\begin{array}{c}\text { Total } \\
(\boldsymbol{n})\end{array}$ & $\begin{array}{c}\text { No } \\
\text { Complications } \\
(\boldsymbol{n}[\%])\end{array}$ & $\begin{array}{c}\text { Thromboembolic } \\
\text { Complication } \\
(\boldsymbol{n}[\%])\end{array}$ & $\begin{array}{c}\boldsymbol{P} \\
\text { Value }\end{array}$ \\
\hline $\begin{array}{l}\text { VerifyNow } \\
\text { Resistant }\end{array}$ & 52 & 46 & 88.4 & 6 & 11.5 & .6413 \\
$\quad \begin{array}{l}\text { Responsive } \\
\text { Multiplate }\end{array}$ & 51 & 48 & 94.1 & 3 & 5.9 & \\
$\quad$ Resistant & 35 & 31 & 88.6 & 4 & 11.4 & .5816 \\
$\quad$ Responsive & 68 & 63 & 92.7 & 5 & 7.5 & \\
$\begin{array}{l}\text { LTA } \\
\text { Resistant }\end{array}$ & 49 & 42 & 85.7 & 7 & 14.3 & .0938 \\
$\quad$ Responsive & 54 & 52 & 96.3 & 2 & 3.7 & \\
\hline
\end{tabular}

Table 3: Relationship of clopidogrel resistance and thromboembolic complications

\begin{tabular}{lcccc}
\hline Method & $\begin{array}{c}\text { Sensitivity } \\
\text { (\%) }\end{array}$ & $\begin{array}{c}\text { Specificity } \\
(\%)\end{array}$ & $\begin{array}{c}\text { Positive } \\
\text { Predictive } \\
\text { Value (\%) }\end{array}$ & $\begin{array}{c}\text { Negative } \\
\text { Predictive } \\
\text { Value (\%) }\end{array}$ \\
\hline VerifyNow & 66.7 & 51.1 & 11.5 & 94.1 \\
Multiplate & 44.4 & 67.0 & 11.4 & 92.7 \\
LTA & 77.8 & 55.3 & 14.3 & 96.3 \\
\hline
\end{tabular}

fers between clopidogrel and the newer generations of antiplatelet agents such as the thienopyridine prasugrel. The conversion of prasugrel to its active metabolite involves cytochrome P450 subenzymes different than those involved by clopidogrel. The cyclopentyltriazolopyrimidine ticagrelor is not a prodrug and does not need cytochrome P450-dependent conversion at all. In contrast to clopidogrel, the common functional cytochrome $\mathrm{P} 450$ genetic variants did not affect active drug metabolite levels and platelet inhibition in patients treated with prasugrel in a cardiology study. The complication rate in percutaneous coronary intervention was not increased by any kind of cytochrome P450 gene variation in patients who were treated with prasugrel. ${ }^{11}$

Because of the increased thromboembolic risk and unreliable test results for clopidogrel resistance, neurointerventionists have discussed the alternative application of these new antiplatelet agents. However, in patients with acute coronary syndromes and a history of stroke or TIA who were monitored over a time period of 15 months, the rate of intracranial bleeding was found to be $2.3 \%$ among patients on aspirin and prasugrel compared with $0 \%$ in the aspirin-plus-clopidogrel arm. ${ }^{12}$ According to this study, prasugrel is contraindicated in patients with recent stroke or TIA and cannot be used as an alternative to clopidogrel as a periprocedural stent-placement medication for symptomatic extracranial or intracranial stenosis, regularly associated with TIA or stroke. In another recent study in which aspirin plus ticagrelor versus aspirin plus clopidogrel were compared in patients with acute coronary syndromes, a previous history of ischemic stroke or TIA was identified as a significant risk factor for impaired clinical outcome, but the bleeding rates in these high-risk patients who received ticagrelor were consistent with those of the overall trial population, and a favorable clinical benefit was found. ${ }^{13}$

In neurovascular stent placement, the alternative use of prasugrel or ticagrelor may be indicated for patients with clopidogrel resistance. Pilot studies have found contradictory results. In a study that included 16 patients, prasugrel was found to be effective and safe for neurointerventional procedures. ${ }^{14}$ In another study that included 67 patients in various clinical conditions, including intracranial bleeding caused by arteriovenous malformation and aneurysm rupture, the use of aspirin and prasugrel in clopidogrel-resistant patients was associated with an increased risk of hemorrhagic complications over that of aspirin and clopidogrel therapy (19.4\% vs $3.6 \%$, respectively; $P=.02) .{ }^{15}$ Additional studies in the setting of neurovascular stent placement with these alternative drug administrations and homogeneous study conditions, including postprocedural platelet-inhibition testing, are required.

Dose elevation to $150 \mathrm{mg}$ of clopidogrel daily may be a treatment alternative. In a recent study, the stroke rate after dose adjustment in clopidogrel-resistant patients declined to $4.5 \%$ compared with the $10.3 \%$ stroke rate in those who received standard clopidogrel treatment. ${ }^{5}$ In our study, preprocedural clopidogrel treatment consisted of the standard 75-mg dosage. As a practical approach to preventing delayed complications, the study protocol dictated an increased dosage of $150 \mathrm{mg}$ of clopidogrel after the procedure if clopidogrel resistance was evident in at least 2 of the 3 assays. Thirty-nine patients were treated with $150 \mathrm{mg}$ of clopidogrel daily for 2 months after extracranial stent placement or for 3 months after intracranial stent placement. Follow-up plateletinhibition testing was not performed. Three patients suffered recurrent ipsilateral TIAs despite the increased clopidogrel dosage during the follow-up period. Only one patient suffered epistaxis and required embolization treatment. Neither intracranial bleeding complications nor stent thrombosis occurred during the 3-month follow-up period. These observations provide a hint that a regimen of $150 \mathrm{mg}$ of clopidogrel daily does not increase the bleeding risk but may not be effective in preventing delayed ischemic complications. However, our study was focused on periprocedural complications, and the follow-up data are not sufficient to draw any conclusions. Additional follow-up studies with alternative medication and continuous platelet-reaction measurements are needed.

There are several limitations to this study. Thromboembolic complications cannot be attributed only to insufficient platelet inhibition by clopidogrel but are the result of various risk factors, such as the degree of atherosclerosis, the configuration of the neurovascular access, the experience of the neurointerventionist, and low-aspirin-responder status. These factors were not analyzed separately in our study. In addition, we included different types of neurovascular stent-placement procedures with different risk profiles, such as stent placement for extracranial and intracranial stenoses and intracranial stent-assisted aneurysm treatment. A control group did not exist. Clopidogrel dosages were not completely homogeneous in the patient cohort, and postprocedural platelet-inhibition testing was not performed.

The strengths of our analysis were the consecutive assessment of all patients, the treatment by only 2 experienced neurointerventionists, and the comparison of 3 different test methods.

\section{CONCLUSIONS}

Clopidogrel resistance is a frequent finding in the clinical context of neurovascular stent placement and seems to be associated with an increased risk of thromboembolic complications. Different testing methods correlate poorly with regard to quantitative and 
qualitative test results. LTA showed a better correlation between clopidogrel resistance and thromboembolic complications than the VerifyNow and Multiplate point-of-care methods.

Additional study with alternative periprocedural antiplatelet drug management involving prasugrel or ticagrelor and close clinical and continuous laboratory monitoring in neurointerventional procedures is warranted.

\section{ACKNOWLEDGMENTS}

We thank Peter Wohlmuth, Asklepios Proresearch, for statistical assistance.

Disclosures: Joachim Röther-UNRELATED: Consultancy: Boehringer Ingelheim, Pfizer, Bristol-Myers Squibb, Bayer, and Lundbeck; Payment for Lectures (including service on speakers bureaus): Boehringer Ingelheim, Pfizer, Bristol-Myers Squibb, Bayer, and Lundbeck. Bernd Eckert-UNRELATED: Payment for Lectures (including service on speakers bureaus): Codman and Covidien, Comments: travel expenses and honoraria for presentations to Congress or during workshops concerning neurointerventional therapies.

\section{REFERENCES}

1. Müller-Schunk S, Linn J. Monitoring of clopidogrel-related platelet inhibition: correlation of nonresponse with clinical outcome in supra-aortic stenting. AJNR Am J Neuroradiol 2008;29:786-91 CrossRef Medline

2. Prabhakaran S, Wells KR, Lee VH, et al. Prevalence and risk factors for aspirin and clopidogrel resistance in cerebrovascular stenting. AJNR Am J Neuroradiol 2008;29:281-85 CrossRef Medline

3. Lee DH, Arat A, Morsi H, et al. Dual antiplatelet therapy monitoring for neurointerventional procedures using a point-of-care platelet function test: a single-center experience. AJNR Am J Neuroradiol 2008;29:1389-94 CrossRef Medline

4. Kang HS, Kwon BJ, Kim JE, et al. Preinterventional clopidogrel response variability for coil embolization of intracranial aneurysms: clinical implications. AJNR Am J Neuroradiol 2010;31:1206-10 CrossRef Medline

5. Fifi JT, Brockington C. Clopidogrel resistance is associated with thromboembolic complications in patients undergoing neurovas- cular stenting. AJNR Am J Neuroradiol 2013;34:716-20 CrossRef Medline

6. Hussein HM, Emiru T, Georgiadis AL, et al. Assessment of platelet inhibition by point-of-care testing in neuroendovascular procedures. AJNR Am J Neuroradiol 2013;34:700-06 CrossRef Medline

7. Gaglia MA, Torguson R, Pakala R, et al. Correlation between light transmission aggregometry, VerifyNow P2Y12, and VASP-P platelet reactivity assays following percutaneous coronary intervention. J Interv Cardiol 2011;24:529-34 CrossRef Medline

8. Kim B, Kim K, Jeon P, et al. Thromboembolic complications in patients with clopidogrel resistance after coil embolization for unruptured intracranial aneurysms. AJNR Am J Neuroradiol 2014;35: 1786-92 CrossRef Medline

9. Brandt JT, Close SL, Iturria SJ, et al. Common polymorphisms of CYP2C19 and CYP2C9 affect the pharmacokinetic and pharmacodynamic response to clopidogrel but not prasugrel. J Thromb Haemost 2007;5:2429-36 CrossRef Medline

10. Mega JL, Hochholzer W, Frelinger AL 3rd, et al. Dosing clopidogrel based on CYP2C19 genotype and the effect on platelet reactivity in patients with stable cardiovascular disease. JAMA 2011;306: 2221-28 CrossRef Medline

11. Mega JL, Close SL, Wiviott SD, et al. Cytochrome P450 genetic polymorphisms and the response to prasugrel: relationship to pharmacokinetic, pharmacodynamic, and clinical outcomes. Circulation 2009;119:2553-60 CrossRef Medline

12. Wiviott SD, Braunwald E, McCabe CH, et al; TRITON-TIMI 38 Investigators. Prasugrel versus clopidogrel in patients with acute coronary syndromes. N Engl J Med 2007;357:2001-15 CrossRef Medline

13. James SK, Storey RF, Khurmi NS, et al; PLATO Study Group. Ticagrelor versus clopidogrel in patients with acute coronary syndromes and a history of stroke or transient ischemic attack. $\mathrm{Circu}$ lation 2012;125:2914-21 CrossRef Medline

14. Stetler WR, Chaudhary N, Thompson BG, et al. Prasugrel is effective and safe for neurointerventional procedures. J Neurointerv Surg 2013;5:332-36 CrossRef Medline

15. Akbari SH, Reynolds MR, Kadkhodayan Y, et al. Hemorrhagic complications after prasugrel (Effient) therapy for vascular neurointerventional procedures. J Neurointerv Surg 2013;5:337-43 CrossRef Medline 